\title{
Attentional changes in blocking are not a consequence of lateral inhibition
}

\author{
Oren Griffiths \\ University of New South Wales, Sydney, Australia \\ AND \\ M. E. Le Pelley \\ Cardiff University, Cardiff, Wales
}

\begin{abstract}
In three human causal learning experiments, we examined attentional modulation in the blocking task, in which participants typically learn little about a novel cue B when it is paired with a previously trained, predictive cue A. Evidence indicates that this blocking training led to a decrement in attention to the blocked cue B. The present experiments addressed whether this decrease in attention to the blocked cue could be better explained as being due to lateral inhibition from the pretrained cue A to the blocked cue B, or as a cue-specific property that is not conditional on the presence or absence of other stimuli. Strong effects of learned predictiveness were observed on participants' causal judgments (Experiment 1) and choice behavior (Experiments 2 and 3). However, no evidence for lateral inhibitory processes emerged in any of the experiments, despite explicit attempts to maximize experimental sensitivity to this effect. The results are discussed in the context of formal models of the operation of attentional processes in human and animal learning.
\end{abstract}

\begin{abstract}
A key issue in the field of associative learning relates to understanding the mechanisms of cue competition: Learning about simultaneously presented cues does not proceed independently for each cue; rather, the cues interact in the learning process. One particular instantiation of cue competition, the blocking effect (Kamin, 1969), has been the focus of much research since its discovery nearly four decades ago. In a typical blocking procedure, participants receive training, in which cue $\mathrm{A}$ is paired with the outcome $(\mathrm{A}+$ trials $)$, prior to receiving trials on which cues $\mathrm{A}$ and $\mathrm{B}$ are presented in compound and paired with the outcome (AB+ trials). Under these circumstances, participants typically learn little about the relationship between cue $\mathrm{B}$ and the outcome; learning about cue $\mathrm{B}$ is said to be blocked by participants' pretraining with cue $\mathrm{A}$.

There exist a number of distinct theoretical accounts of blocking, and it has been argued that the phenomenon may be multiply determined (e.g., Holland \& Kenmuir, 2005; Le Pelley, 2004). Evidence from studies of animal conditioning (Mackintosh, 1978; Mackintosh \& Turner, 1971) and human learning (Kruschke \& Blair, 2000; Le Pelley, Beesley, \& Suret, 2007) indicates that one determinant of blocking is a reduction in the processing of the blocked cue. Specifically, these studies demonstrated that, following a blocking procedure, rats and humans were slow to learn a new association between the previously blocked cue and a novel outcome. For example, Kruschke and Blair (2000) used a blocking procedure in which human
\end{abstract}

participants were required to predict the presence of several mutually exclusive outcomes (o1, o2, o3, o4, o5, o6). That is, participants were initially given pretraining, in which cue A was paired with outcome ol (A-ol trials); they were then presented compound trials, in which cues $A$ and $B$ were presented together and paired with the same outcome $(\mathrm{AB}-\mathrm{O} 1$ trials). During this second training phase, participants also experienced HI-06 control trials, for which neither element had received pretraining. When subsequently presented with the compound $\mathrm{BH}$ during a test phase, participants indicated that outcome o6 was more likely to occur than was outcome 01 , indicating that the $\mathrm{B}-\mathrm{o} 1$ association was weaker than the $\mathrm{H}-\mathrm{o} 6$ association. That is, blocking had occurred.

This blocking treatment was followed by a third phase of training, which included $\mathrm{ABC}-\mathrm{O} 2$ trials - that is, trials on which the previously blocked cue (B) was paired with a novel outcome. Participants also experienced DEF-o4 control trials, for which cues $\mathrm{E}$ and $\mathrm{F}$ were novel and, hence, had not been previously blocked (cue D had been consistently and individually paired with outcome 03 during prior training). When participants were subsequently presented compound $\mathrm{BE}$ during a final test phase, they indicated that outcome o4 was more likely to occur than was outcome $\mathrm{o} 2$, indicating that the $\mathrm{E}-\mathrm{o} 4$ association was stronger than the $\mathrm{B}-\mathrm{O} 2$ association. In other words, participants learned less about the previously blocked cue B than about the control cue $\mathrm{E}$, which had received identical 
treatment during this third phase of training, but had not previously been blocked. These results indicate that the initial blocking procedure had led to a reduction in the rate of learning about the blocked cue B in a subsequent learning task, relative to a control cue that had not been blocked.

This finding is predicted by certain associative models that allow for learned changes in the processing of cuesthat is, changes in the extent to which a cue is able to engage the learning process (e.g., Kruschke, 2001, 2006; Mackintosh, 1975; Pearce \& Hall, 1980). This ability to engage the learning process is often described as being related to the attention paid to the cue or the associability of that cue, denoted as $\alpha$. The distinction between attention and associability is unimportant for the present analysis; hence, we will use the terms interchangeably here (for a discussion of this distinction, see Dickinson \& Mackintosh, 1979). These models propose that the $\alpha$ value of each cue is a function of the experienced predictiveness of that cue. The Mackintosh and Kruschke models suggest that $\alpha$ increases for the most predictive cue present and decreases for all others. (The Pearce-Hall model takes a rather different approach and is discussed further in the General Discussion.) Therefore, these models predict that during a blocking task, attention to the blocked cue $\mathrm{B}$ will be reduced, because $B$ is a poorer predictor of the outcome on $\mathrm{AB}-\mathrm{o} 1$ trials than is $\mathrm{A}$, as a consequence of the pretraining of A. Consequently, B's ability to engage the learning process is reduced; hence, little is learned about $\mathrm{B}$ on these $\mathrm{AB}-\mathrm{O} 1$ trials. Moreover, the suggestion that blocking treatment leads to a decline in $\alpha_{\mathrm{B}}$ also allows these models to account for retardation in subsequent learning about the relationship between B and a novel outcome; such retardation was observed by Kruschke and Blair (2000; see also Le Pelley et al., 2007). Put informally, as a consequence of the prior blocking treatment, participants learned to disregard the blocked cue B and continued to do so during subsequent training.

If it is accepted that these human studies (and their animal conditioning analogues) establish a role for attentional mechanisms in blocking, the next question becomes one of how these mechanisms ought to be implemented in models of learning. Two possibilities arise that are consistent with the existing evidence. The first is that attentional changes are conditional on the presence of other cues, a mechanism that has been termed lateral inhibition (Kruschke, 2006), wherein competing cues can suppress the attention allocated to their competitors. Applying this account to Kruschke and Blair's (2000) blocking study, during $\mathrm{AB}-\mathrm{O} 1$ trials, the presence of the pretrained cue $\mathrm{A}$ will suppress attention to the rival cue $\mathrm{B}$. During subsequent $\mathrm{ABC}-\mathrm{O} 2$ training, the continued presence of $\mathrm{A}$ will continue to suppress attention to $\mathrm{B}$; hence, little will be learned about $\mathrm{B}$, as indicated by the empirical data.

To reiterate, this lateral inhibition view of attention assumes that the suppression of attention to a blocked cue is conditional on the presence of the blocking cue. That is, during the $\mathrm{AB}+$ trials of a blocking procedure, participants learn to ignore cue $\mathrm{B}$ when cue $\mathrm{B}$ is presented in compound with cue A. Were B to be presented alone (or in compound with a cue other than A) following blocking training, it would no longer be subject to the suppression caused by the presence of cue A; hence, attention to B would be restored. This type of lateral inhibition mechanism forms a central part of the attentional process in several of Kruschke's models (e.g., Kruschke, 2001, 2006). Essentially, these models "learn" to distribute attention between the elements that make up a specific stimulus compound.

An alternative possibility is that changes in the distribution of attention are not conditional on specific cue combinations. On this approach, $\alpha$ values "belong" to individual cues and are independent of any other cues that are present on a given trial. Thus, the decline in $\alpha_{\mathrm{B}}$ resulting from the $\mathrm{AB}+$ trials of a blocking procedure will result in slower subsequent learning about $\mathrm{B}$, regardless of whether it is subsequently trained in the presence or absence of A. This unconditional view of attentional changes is that taken by Mackintosh's (1975) model, among others (e.g., Le Pelley, 2004).

A third possibility is that attention is influenced by both conditional and unconditional mechanisms. This is the approach taken, for example, by Kruschke's (2006) locally Bayesian model of attention in learning. In this model, attention is determined by the strength of a cue's representation in the "hidden" layer of a learning system, where the mapping from cue to outcome is mediated. The strength of this representation can be influenced by lateral inhibition from other cues (a conditional component of attention) and by the strength of the direct association from the input representation of that cue to its own hidden unit representation (an unconditional component of attention). This two-mechanism approach increases the flexibility of the resulting model: By varying parameters, such models can behave in a way similar to purely unconditional models, purely conditional models, or a mixture of the two. Of course, this increase in flexibility comes at a cost, in terms of parsimony.

The question therefore becomes one of whether it is necessary to invoke both conditional and unconditional mechanisms controlling attention, or whether a single mechanism is sufficient. This question underlies the present experiments, in which we tested the conditionality of attention by using a procedure similar to that of Le Pelley et al. (2007). In each experiment, participants initially experienced a blocking procedure (e.g., $\mathrm{A}-\mathrm{o} 1$, then $\mathrm{AB}-\mathrm{o} 1$ ). Following this, in a final training phase, the same set of cues was presented to participants, but the participants were required to learn about a different set of outcomes. That is, participants were required to engage in new learning about the previously trained cues. It is important to note that during this final training phase, participants were presented a series of cue compounds that each consisted of one cue that had previously been blocked and one cue that had previously been a blocking cue. Half of these cue compounds were identical to the blocking compounds that were presented during the second training phase (e.g., $\mathrm{AB}-\mathrm{O} 3$ trials). This is similar to the procedure used in Kruschke and Blair's (2000) study. Both conditional and unconditional views of attention predict that participants will attend less to the previously blocked cues (e.g., cue B) in these compounds and will, consequently, learn less about 
them. Other compounds in this final training phase also each contained one previously blocked cue and one former blocking cue. However, these cues had not previously appeared in combination with one another. The conditional view of attention predicts that presenting these previously blocked cues in novel compounds (and crucially in the absence of the specific cues that blocked them) will release them from suppression and, hence, restore attention to them, relative to the blocked cues that were presented in their original blocking compounds throughout training. In contrast, the unconditional view of attention states that attentional changes are not specific to particular stimulus compounds, so whether the blocked cue is presented in its original blocking compound or in a novel compound will have no influence on the attention paid to it or on the amount learned about it. In line with this view, a similar decrement in learning will be seen for blocked cues, regardless of the cues with which they are paired.

\section{EXPERIMENT 1}

The design of Experiment 1 is shown in Table 1. This experiment used an allergist task (Dickinson \& Burke, 1996), in which each participant assumed the role of an allergist looking at the causes of allergic reactions in two fictitious patients, Mr. X and Mr. Y. In this paradigm, the cues $\mathrm{A}$ through $\mathrm{X}$ were represented by different foods, and outcomes o1 to 04 were represented by different types of allergic reactions. Thus, Mr. X and Mr. Y ate the same foods, but they suffered from mutually exclusive sets of allergic reactions. The participants' task was to learn which type of allergic reaction would occur following each meal.

During Phases 1 and 2, participants learned about foods and outcomes for Mr. X. These two phases included six separate blocking treatments (labeled "blocking contin-

Table 1

Design of Experiment 1

\begin{tabular}{|c|c|c|c|}
\hline \multicolumn{2}{|c|}{ Mr. X } & \multicolumn{2}{|c|}{ Mr. Y } \\
\hline Phase 1 & Phase 2 & Phase 3 & Test \\
\hline \multicolumn{4}{|c|}{ Blocking Contingencies } \\
\hline $\mathrm{A}-\mathrm{o} 1$ & $\mathrm{AB}-\mathrm{ol}$ & AB-o3 (old) & A, B \\
\hline $\mathrm{C}-\mathrm{o} 1$ & $\mathrm{CD}-\mathrm{o} 1$ & ED-o3 (new) & E, D \\
\hline $\mathrm{E}-\mathrm{o} 1$ & $\mathrm{EF}-\mathrm{O} 1$ & & \\
\hline $\mathrm{G}-\mathrm{o} 2$ & $\mathrm{GH}-\mathrm{O} 2$ & GH-O4 (old) & $\mathrm{G}, \mathrm{H}$ \\
\hline $\mathrm{I}-\mathrm{O} 2$ & $\mathrm{IJ}-\mathrm{o} 2$ & $\mathrm{KJ}-\mathrm{O} 4$ (new) & $\mathrm{J}, \mathrm{K}$ \\
\hline $\mathrm{K}-\mathrm{o} 2$ & $\mathrm{KL}-\mathrm{o} 2$ & & \\
\hline \multicolumn{4}{|c|}{ Change Contingencies } \\
\hline $\mathrm{M}-\mathrm{o} 1$ & $\mathrm{MN}-\mathrm{O} 2$ & $\mathrm{MN}-\mathrm{O} 3$ (old) & $\mathrm{M}, \mathrm{N}$ \\
\hline $\mathrm{O}-\mathrm{o} 1$ & $\mathrm{OP}-02$ & $\mathrm{QP}-03$ (new) & $\mathrm{Q}, \mathrm{P}$ \\
\hline $\mathrm{Q}-\mathrm{o} 1$ & $\mathrm{QR}-\mathrm{0} 2$ & & \\
\hline $\mathrm{S}-\mathrm{o} 2$ & ST-o1 & ST-o4 (old) & $\mathrm{S}, \mathrm{T}$ \\
\hline $\mathrm{U}-\mathrm{o} 2$ & UV-o1 & WV-o4 (new) & $\mathrm{W}, \mathrm{V}$ \\
\hline $\mathrm{W}-\mathrm{o} 2$ & WX-o1 & & \\
\hline
\end{tabular}

Note $-\mathrm{A}$ through $\mathrm{X}$ represent foods, and o1 through 04 indicate allergic reactions. "Old" and "new" refer to the novelty of the compound appearing during Phase 3 of training: Old compounds are those that were previously experienced during Phase 2, and new compounds comprised equivalent elements that had not been paired with each other prior to Phase 3. All participants received all types of trials listed under a given stage of training. gencies" in Table 1). That is, participants were initially trained with individual foods and an outcome (e.g., carrot $\rightarrow$ dizziness), and then in Phase 2 were presented with compound trials, in which the pretrained food (carrot) was paired with both a new food (cheese) and the same allergic reaction (e.g., carrot, cheese $\rightarrow$ dizziness). Both views of attention discussed earlier predict that this blocking training will lead to a decrease in attention to the blocked cue (B, D, F, H, J, L, in Table 1; hereafter referred to as target cues), whereas attention to the blocking cue (A, C, E, G, I, K; hereafter referred to as competing cues) remains high.

During Phase 3, participants were introduced to a new patient, Mr. Y, who suffered two allergic reactions different from Mr. X's. Participants were, as with Mr. X, required to learn which allergic reaction would follow each meal eaten by Mr. Y. Following Le Pelley et al. (2007), the allergic reactions suffered by Mr. Y were statistically independent of those suffered by Mr. X. That is, of the foods that were paired with outcome ol in the Mr. X task, half were paired with outcome 03 and half were paired with outcome 04 in the Mr. Y task. The same was true for foods that were previously paired with outcome $\mathrm{o} 2$.

Two of the Phase 3 trial types (AB-o3 and $\mathrm{GH}-\mathrm{o} 4$, referred to as old compounds) comprised a pair of cues that had previously been paired with each other during the compound training phase of the blocking treatment (i.e., in Phase 2). As noted earlier, conditional and unconditional views of attention both predict that the learning of associations between the competing cues (A and $\mathrm{G}$ ) and their respective outcomes will occur more rapidly than for the target cues ( $\mathrm{B}$ and $\mathrm{H}$ ) and those same outcomes. For example, at the end of Phase 3, the A-o3 association should be stronger than the $\mathrm{B}-\mathrm{o} 3$ association. This effect is analogous to the blocking-of-unblocking effect observed in both human learning (Kruschke \& Blair, 2000; Le Pelley et al., 2007) and animal conditioning (Mackintosh, 1978; Mackintosh \& Turner, 1971), wherein blocked cues are found to be slower than control cues to form novel associations.

The remaining two compounds (ED and KJ, referred to as new compounds) also comprised one competing cue and one target cue, and therefore had the same reinforcement history as the old compounds. However, these pairs of cues were not trained in compound with each other during the earlier blocking procedure. If the target cue is presented in the absence of the specific competing cue that blocked it, then, according to the conditional view of attention, it should be released from the suppression caused by the competing cue, and attention to the target cue should be restored. A similar argument applies to the competing cue: Any influences on attention resulting from its earlier compound training will be removed when it is presented in a novel compound, and attention to the competing cue will also be restored to its original level. This conditional view therefore predicts that for these novel compounds, during Phase 3, there will be no difference in learning between the competing cue and the target cue; for example, there should be no difference in learning about $\mathrm{E}$ and D during Phase 3. Moreover, the conditional view 
predicts that during Phase 3, learning about B (attention to which will be inhibited by its pretrained partner A) will proceed more slowly than learning about $\mathrm{D}$ (which will be released from the lateral inhibition formerly caused by its pretrained partner, C). In contrast, the unconditional view of attention predicts similar effects of prior predictive status, regardless of whether the cues in the Phase 3 compound have been trained together or separately in the past. That is, given that this model predicts $\left(\alpha_{\mathrm{A}}=\alpha_{\mathrm{E}}\right)>$ $\left(\alpha_{\mathrm{B}}=\alpha_{\mathrm{D}}\right)$ at the outset of Phase 3, there should be an advantage for $\mathrm{A}$ over $\mathrm{B}$ and for $\mathrm{E}$ over $\mathrm{D}$, and there should be no difference in the size of this advantage for cues A and $\mathrm{E}$ over their partners. Moreover, learning about these partners (B and D) should proceed at the same rate.

The change contingencies in the lower half of Table 1 may also test the extent to which attention to a cue is conditional on the presence of other cues. These change contingencies were similar to the blocking contingencies, except that the outcome paired with the compound during Phase 2 was the opposite of that that had been paired with the competing cue during Phase 1. For example, competing cue $\mathrm{M}$ was pretrained with outcome o1, but in Phase 2, compound MN was paired with outcome 02 . Given that target cue $\mathrm{N}$ is a better predictor of 02 than is $\mathrm{M}$, attention to $\mathrm{N}$ should be maintained at a high level over these compound trials. On the conditional view of attention, we might also expect $\mathrm{N}$ to come to suppress attention to $\mathrm{M}$ when these cues are presented in compound (for a discussion of attention shifting in the highlighting effect, see Kruschke, 2006). Consequently, when the old MN compound is paired with a novel outcome in Phase 3, this account anticipates more rapid learning about the target cue $\mathrm{N}$ than about the competing cue $\mathrm{M}$; a similar argument applies for compound ST. The remaining Phase 3 compounds (QP and WV) comprised an equivalent target and competing cue, but these cues had not previously been paired with one another. Once again, the conditional view of attention anticipates that presenting these cues in novel compounds will release them from any attentional changes that they might have undergone during Phase 2. Hence, this view predicts that, unlike for the old compounds, Phase 3 learning about the target and competing cues from the new compounds of the change contingencies (QP and WV) will proceed at a similar rate.

The predictions made by the unconditional view of attention for the change contingencies are less definite. $\alpha_{\mathrm{M}}$ could be expected to increase during $\mathrm{M}-\mathrm{O} 1$ pretraining in Phase 1 of an $\mathrm{M}-\mathrm{o} / \mathrm{MN}-\mathrm{o} 2$ contingency, and this initial increase will tend to offset any reduction in $\alpha_{\mathrm{M}}$ that occurs on $\mathrm{MN}-\mathrm{O} 2$ trials. In addition, interspersed throughout Phase 2 were reminder blocks of Phase 1 trials (see the Procedure). Thus, $\mathrm{M}-\mathrm{O} 1$ trials would occasionally occur during $\mathrm{MN}-\mathrm{O} 2$ compound training, further counteracting any reduction in $\alpha_{\mathrm{M}} \cdot{ }^{1}$ Consequently, the predictions of the unconditional model for these change contingencies critically depend on the rate at which $\alpha_{\mathrm{M}}$ increases, relative to the rate at which it decreases. Typically, these rates are not specified in unconditional attentional models (e.g., Mackintosh, 1975), and thus it is possible that $\alpha_{\mathrm{M}}$ might begin Phase 3 lower than, higher than, or at a similar level to, $\alpha_{\mathrm{N}}$.
Therefore, this account makes no clear prediction regarding the relative rate of learning about target and competing cues during Phase 3. It does, however, predict that any differences between the target and competing cues will be equivalent for old compounds (MN and ST) and new compounds (QP and WV).

\section{Method}

Participants and Stimuli. Forty-eight Cardiff University undergraduates participated for course credit. The following 24 foods were randomly and independently assigned to cues $\mathrm{A}-\mathrm{X}$ in Table 1 for each participant: garlic, yogurt, peaches, noodles, onion, banana, bread, grapes, pasta, eggs, chicken, mushrooms, carrots, tomato, lettuce, ham, rice, potato, apples, cucumber, steak, spinach, oranges, and tuna. Two easily differentiable line drawings of men were used to represent Mr. X and Mr. Y. Microsoft Paint was used to alter these images to depict each of the allergic reaction outcomes (nausea, dizziness, itchiness, headache). These reactions were randomly assigned to outcomes o1 to o4 for each participant.

Procedure. Participants were instructed that they were to play the role of an allergist, investigating a patient, Mr. X, who had eaten a series of different foods on different days and experienced certain allergic reactions on those days. Participants were informed that their task was to learn which foods caused Mr. X to experience those reactions and that they would later be asked questions about those foods and reactions.

Following the instructions, participants were presented the names of one or two foods onscreen for each trial. Participants were required to predict which reaction $\mathrm{Mr}$. X would experience, by clicking on one of two onscreen response buttons labeled with the names of outcomes o1 and o2, respectively (e.g., dizziness and itchiness). These buttons were separated horizontally: The outcome ol response button was positioned to the left of the outcome 02 response button. Feedback was then provided, along with an image of Mr. X experiencing one of the symptoms. When participants predicted the incorrect reaction, a tone sounded, and the screen that displayed the cues and the correct outcome froze for $2 \mathrm{sec}$ before the participants were able to progress to the next trial. Phase 1 comprised 10 blocks, with each of the 12 trial types shown in Table 1 appearing in random order once per block. Phase 2 immediately followed Phase 1 (with no break) and comprised 8 blocks, again with each of the 12 trial types appearing once per block. Participants were given 2 reminder blocks of Phase 1 trials - one after the 2nd and 6th blocks of Phase 2 training.

Following Phase 2 training, participants received more instructions, which explained that they were now required to learn about a new patient, Mr. Y, and that they might be asked questions about $\mathrm{Mr}$. Y at the end of training. Participants received 5 blocks of the Phase 3 training detailed in Table 1. Phase 3 training differed from Phase 2 in two ways: (1) The horizontally separated response buttons for outcomes 01 and 02 were removed, and two new response buttons, labeled with outcomes 03 and o4, were provided. To emphasize the independence of the new outcomes ( 03 and 04 ), the new response buttons were vertically separated: The outcome 03 response button was positioned above the outcome 04 response button; and (2) the picture used to illustrate the correct answer on each trial showed the Mr. Y figure, rather than the Mr. X figure.

On completion of training, participants moved to a test phase, in which they were presented a single food and were asked to rate how likely it was that that food would cause a given reaction in Mr. Y. Ratings were provided on a scroll bar with a minimum value of 0 (indicating that the food was very unlikely to cause that type of allergic reaction) and a maximum of 100 (indicating that the food was very likely to cause that type of reaction). Each of the 16 foods presented during the Mr. Y task was rated twice in the test phase: once for how strongly it predicted outcome 03 , and (on the immediately succeeding trial) once for how strongly it predicted outcome 04 . The test trial order was randomized for each participant. 


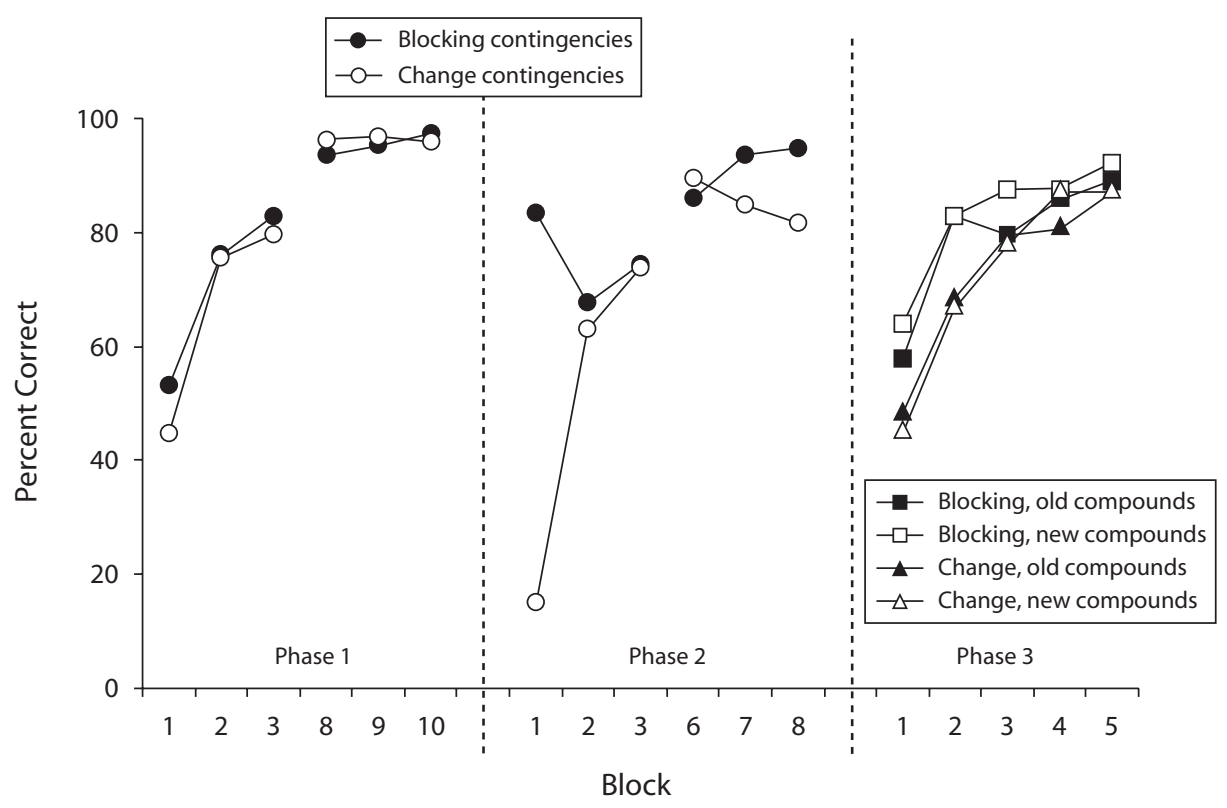

Figure 1. Mean percent correct responses for Experiment 1, showing the first and last three blocks of Phases 1 and 2, and all five blocks of Phase 3. For Phases 1 and 2, data are averaged separately over all blocking and change contingencies. For Phase 3, data are shown separately for old and new compounds for the blocking and change contingencies.

\section{Results}

Participants could be expected to show an influence of prior learning on the rate at which subsequent learning occurred only if they were able to learn the cue-outcome contingencies of this experiment. A relatively liberal performance criterion was adopted, whereby any participant failing to perform above chance (50\%) averaged over the final 5 blocks of any phase was excluded. This criterion resulted in the removal of 16 participants. The training data for the remaining 32 participants are plotted in Figure 1. Across each of the three training phases, participants showed good acquisition of the presented contingencies.

The data of most interest relate to participants' learning of the relationships between the foods and the specific reactions with which the foods were paired during Phase 3-that is, not how strongly each food was perceived to cause reactions in general, but, instead, how strongly it was perceived to cause the reaction with which it had been paired, as opposed to causing the reaction with which it was not paired. This indicates the participants' selective learning about each cue. Following Le Pelley et al. (2007; see also Le Pelley, Oakeshott, \& McLaren, 2005), difference scores for each cue were computed by taking each participant's ratings of causation for the outcome with which that cue was paired during Phase 3 training (the correct outcome), and subtracting from this value their ratings of causation for the outcome that was not paired with that cue during training (the incorrect outcome). For example, the difference score for cue A was calculated by taking the rating for cue A with respect to outcome $\mathrm{o} 3$ (because A was paired with 03 in Phase 3), and subtracting from that the rating for cue $\mathrm{A}$ with respect to outcome 04 (because A was not paired with o4 in Phase 3). A high difference score $($ maximum $=100)$ indicated selective, accurate ratings of causation, and a score of 0 indicated chance performance.

Participants' difference scores are displayed in Figure 2. These scores have been averaged separately for cues trained in old compounds versus new compounds during Phase 3, and for target cues versus competing cues. Figure 2A shows scores for cues from the blocking contingencies; Figure 2B shows scores for those from the change contingencies.

The data for the blocking contingencies were analyzed using a repeated measures ANOVA with factors of cue type (target cue vs. competing cue) and compound (old vs. new). Significance in this and all subsequent analyses was assessed against a Type I error rate of $\alpha=.05$. This analysis revealed a significant main effect of cue type $[F(1,31)=6.82]$, with target cues receiving significantly lower ratings than competing cues. Crucially, the interaction between cue type and compound was nonsignificant $(F<1)$, indicating that the magnitude of the difference between target and competing cues did not depend on whether those cues were trained in old or new compounds during Phase 3. Figure 2A also reveals that in general, participants' ratings for cues trained in old compounds tended to be higher than for cues that were trained in new compounds. This difference, assessed by the main effect of compound, approached significance $[F(1,31)=3.56$, $p=.07]$. Follow-up, planned $t$ tests revealed that the target cues were significantly less well learned about than were the competing cues, both when presented in the same compounds during Phases 2 and $3[t(31)=2.23]$ and when they were presented in novel compounds in Phase 3 $[t(31)=2.07]$. Finally, a planned $t$ test revealed that there 

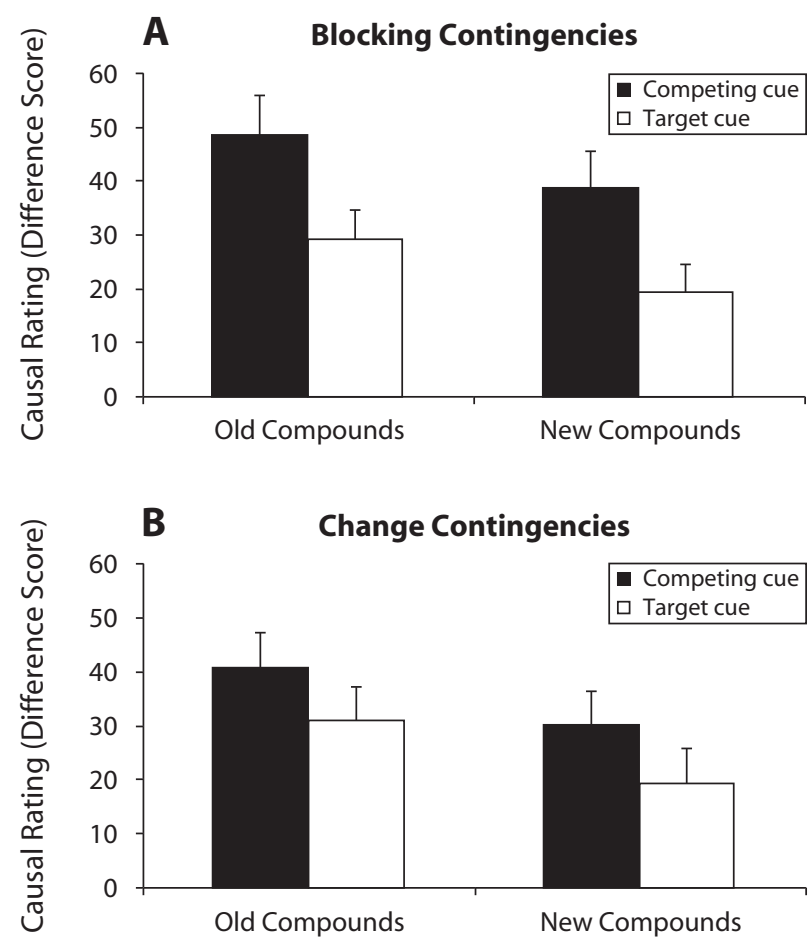

Figure 2. Mean difference scores for the test cues of Experiment 1 . Difference scores were calculated by subtracting the causal judgment rating for the allergy with which the cue was not paired in Phase 3 from the rating for the allergy with which it was paired. (A) Scores for blocking contingencies. (B) Scores for change contingencies.

was no significant difference in the difference scores for target cues from old or new compounds $[t(30)=1.44]$.

Turning to the change contingencies, Figure $2 \mathrm{~B}$ indicates that learning was, if anything, generally poorer for the target cues than for the competing cues. However, an ANOVA equivalent to that described above revealed that the main effect of cue type failed to reach significance $[F(1,31)=1.57]$. Consistent with the results of the blocking contingencies, ratings for cues trained in old compounds during Phase 3 were generally higher than for cues trained in novel compounds: For the change contingencies, there was a significant main effect of compound $[F(1,31)=5.27]$. Finally, the interaction of cue type with compound was nonsignificant $(F<1)$, indicating that the pattern of relative responding to target and competing cues did not differ for cues trained in old and new compounds.

\section{Discussion}

Results from the blocking contingencies demonstrated a significant learned predictiveness effect: Learning during Phase 3 was modulated by the prior predictiveness of the cues. Specifically, participants were slower to learn a new relationship involving a previously blocked target cue than they were a pretrained competing cue, replicating the findings of Kruschke and Blair (2000) and Le Pelley et al. (2007). This result clearly indicates that the paradigm used in Experiment 1 was sensitive to the influence of prior learning on cue processing, legitimizing it as a means of assessing the conditionality of these changes in cue processing.

Critically, the new/old status of the training compounds did not significantly alter the magnitude of this learned predictiveness effect. In particular, the amount learned about a previously blocked target cue during Phase 3 did not depend on whether that cue was trained in an old or a new compound. These findings run contrary to the conditional view of attention, which suggests that the root of the decline in attention to a blocked cue is lateral inhibition from the blocking cue with which it was trained. Such a view is constrained to predict that for novel Phase 3 compounds, learning will progress at an equal rate for target and competing cues, yet a significant difference in responding to these cues was observed. The results are instead consistent with the unconditional view of attention taken, for example, by Mackintosh (1975), in which $\alpha$ is a property that "belongs to" a cue and is not directly determined by the presence or absence of specific competing cues.

Results from the change contingencies were somewhat less conclusive. As noted earlier, the conditional view of attention anticipates that the presence of the target cue (e.g., N) should come to suppress attention to the competing cue (e.g., M) during Phase 2, which should lead to greater learning about target cues than about competing cues trained in old compounds during Phase 3. As we found previously, this difference between target and competing cues should not occur when they are presented in new compounds. In fact, there was a nonsignificant trend toward greater learning about competing cues than about target cues, and this trend did not interact with the new/ old status of the Phase 3 compound. Thus, as with the data from the blocking contingencies, the data from the change contingencies were not supportive of the conditional account of attention.

As argued earlier, it is possible for the unconditional view to capture this pattern of results. To do so, this account would have to posit that $\alpha$ increases more rapidly than it decreases. Under these parameters, the $\alpha$ value for the competing cue could be expected to begin Phase 3 at a level similar to, or even higher than, that of the target cue. However, in the absence of a significant effect of cue type in these change contingencies, or in the absence of independent evidence regarding the rates at which $\alpha$ increases and decreases, this interpretation must remain tentative.

The results also revealed that in general, cues trained in old compounds during Phase 3 tended to receive higher ratings than did those trained in novel compounds, a trend that approached significance for the blocking contingencies and was significant for the change contingencies. We postpone further discussion of this issue until after Experiment 2.

The finding of a learned predictiveness effect for blocked target cues and pretrained competing cues trained in a novel compound during Phase 3 contradicts the idea that changes in attention are entirely determined by a process of lateral inhibition (and, hence, conditional on the presence of the cues that cause this lateral inhibition). This 
is not entirely surprising, given that prior studies have demonstrated learned predictiveness effects in human contingency learning using novel cue compounds during the critical learning phase (e.g., Le Pelley \& McLaren, 2003; Le Pelley, Oakeshott, Wills, \& McLaren, 2005), and these include studies of learned predictiveness effects in blocking (Le Pelley et al., 2007). The present work extends and strengthens the conclusions that can be drawn from these earlier studies, by providing a direct comparison of the magnitude of learned predictiveness effects in old and new compounds. Specifically, the finding that the difference in learning about target and competing cues from the blocking contingencies was of equal magnitude for old and new compounds implies that lateral inhibition does not exert a significant influence in this paradigm. That is, the decreased attention for the previously blocked target cues in Experiment 1 appeared to be entirely unconditional on the presence of specific competing cues.

This conclusion, however, relies on a null effect (the lack of a difference in learned predictiveness effects for old and new compounds). Perhaps Experiment 1 was simply not sensitive enough to yield evidence of lateral inhibitory processes. This, however, seems unlikely. The difference between target and competing cues trained in old compounds was relatively large (see Figure 2A). Furthermore, neither the target nor competing cues received difference scores that were particularly close to the theoretical minimum (0) or maximum (100) values, respectively. There was, therefore, considerable scope for detecting a difference in the magnitude of this learned predictiveness effect. Nevertheless, it could be argued that the failure to detect a difference in learning between previously blocked target cues trained in old and new compounds was a result of a floor effect. Thus, a second experiment was conducted, in which the training procedure and test measure were altered to maximize the sensitivity of the paradigm and minimize the possibility of floor effects, hence maximizing the chance of observing an effect of lateral inhibition on the blocked target cue.

\section{EXPERIMENT 2}

The design of Experiment 2 (shown in Table 2) was similar to that of Experiment 1. Once again, the Mr. X task comprised a number of simultaneous blocking procedures (in this case, eight), and again, each Phase 3 compound was composed of one former blocking cue (the competing cue) and one previously blocked cue (the target cue). For the old compound conditions, these compounds comprised cues that had been paired with each other during the compound phase of the blocking procedure; for the new compound condition, these compounds comprised cues that had not previously been paired with each other. Given that the change contingencies of Experiment 1 yielded slightly ambiguous results, they were omitted from Experiment 2. That said, Experiment 2 did feature filler trials in Phases 1 and 2 that were similar to the Phase 1 and 2 training of the previous change contingencies. These filler trials were included only to keep the training phases of Experiments 1 and 2 as similar as
Iable 2

Design of Experiment 2

\begin{tabular}{|c|c|c|c|}
\hline \multicolumn{2}{|c|}{ Mr. X } & \multicolumn{2}{|c|}{ Mr. Y } \\
\hline Phase 1 & Phase 2 & Phase 3 & Test \\
\hline \multicolumn{4}{|c|}{ Old Compounds } \\
\hline $\mathrm{A}-\mathrm{o} 1$ & $\mathrm{AB}-\mathrm{o} 1$ & $\mathrm{AB}-\mathrm{o} 3$ & A vs. F \\
\hline $\mathrm{C}-\mathrm{ol}$ & $\mathrm{CD}-\mathrm{o} 1$ & CD-o4 & C vs. H \\
\hline $\mathrm{E}-\mathrm{o} 2$ & $\mathrm{EF}-\mathrm{o} 2$ & $\mathrm{EF}-\mathrm{o} 3$ & E vs. B \\
\hline $\mathrm{G}-\mathrm{o} 2$ & $\mathrm{GH}-\mathrm{o} 2$ & $\mathrm{GH}-\mathrm{o} 4$ & G vs. D \\
\hline \multicolumn{4}{|c|}{ New Compounds } \\
\hline $\mathrm{I}-\mathrm{o} 1$ & $\mathrm{IJ}-\mathrm{o} 1$ & $\mathrm{IL}-\mathrm{o} 3$ & L vs. B \\
\hline $\mathrm{K}-\mathrm{o} 1$ & $\mathrm{KL}-\mathrm{ol}$ & $\mathrm{KJ}-\mathrm{O} 4$ & J vs. D \\
\hline $\mathrm{M}-\mathrm{o} 2$ & $\mathrm{MN}-\mathrm{O} 2$ & $\mathrm{MP}-\mathrm{o} 3$ & P vs. F \\
\hline $\mathrm{O}-\mathrm{o} 2$ & $\mathrm{OP}-\mathrm{o} 2$ & $\mathrm{ON}-\mathrm{o} 4$ & $\mathrm{~N}$ vs. $\mathrm{H}$ \\
\hline \multicolumn{4}{|c|}{ Fillers } \\
\hline $\mathrm{Q}-\mathrm{o} 1$ & $\mathrm{QR}-\mathrm{o} 2$ & & \\
\hline $\mathrm{S}-\mathrm{o} 1$ & $\mathrm{ST}-\mathrm{o} 2$ & & \\
\hline $\mathrm{U}-\mathrm{o} 2$ & UV-o1 & & \\
\hline $\mathrm{W}-\mathrm{o} 2$ & WX-o1 & & \\
\hline
\end{tabular}

possible; therefore, we did not test participants' learning about them. These cues will not be discussed further.

The main difference between Experiments 1 and 2 was that the latter used a forced choice testing procedure. That is, on each test trial, participants were forced to choose which of two foods would more likely cause a particular outcome, thus providing a relative measure of the strength of the association between each cue and that outcome. Whereas comparisons between individual ratings of cues, as used in Experiment 1, include variability in the rating procedure (e.g., participants may become more or less liberal as the testing procedure proceeds), forced choice procedures allow direct and simultaneous comparison between two cues. Furthermore, the procedure may amplify any small differences between cues, because participants are forced to respond maximally, regardless of how unsure they are. Finally, a forced choice testing procedure reduces the potential influence of floor or ceiling effects, again because participants are forced to make a relative judgment, rather than provide ratings on an absolute scale.

Of primary interest are the lower four test trials shown in Table 2, on which participants were forced to choose between two previously blocked target cues: one that was trained in an old compound in Phase 3 (cues B, D, $\mathrm{F}, \mathrm{H}$ ), and one that was trained in a new compound (cues $\mathrm{L}, \mathrm{J}, \mathrm{P}, \mathrm{N})$. The conditional view of attention suggests that presenting target cues in new compounds removes the lateral inhibitory links between the competing cues (e.g., cue I) and target cues (cue L), and will therefore result in better learning for these target cues trained in new compounds than for those trained in the same cue compounds throughout training (e.g., cue B). Hence, for each of these four test trials, this view predicts that participants will consistently pick (as more predictive) the target cue from the new compound over that from the old compound. In contrast, the unconditional view does not predict any differences between cues presented in new or old compounds; hence, participants' choices should be randomly and equally divided between cues from old and new compounds. 
Additionally, for each of the upper four test trials shown in Table 2, participants were forced to choose between target cues from old compounds (cues B, D, F, H) and competing cues from old compounds (cues A, C, E, G). As noted earlier, both conditional and unconditional views of attention predict that, for these old compounds, the target cues will be less well learned about than the competing cues, and therefore will be chosen less often than the competing cues.

\section{Method}

Participants and Stimuli. Experiment 2 was conducted with a new set of participants. Thirty-four University of New South Wales undergraduates participated in exchange for course credit. The stimuli were the same as those for Experiment 1.

Procedure. The training procedure of Experiment 2 was identical to that of Experiment 1, except where already noted. The test phase of Experiment 2 consisted of eight forced choice trials, on which an outcome was presented onscreen, along with two cues. On each test trial, participants were required to select which of the two presented cues was more likely to have caused the presented outcome. The order in which these two foods appeared on the screen (left/right) was randomized on each trial. The outcome presented on each test trial was that which had been paired with both of the presented cues during Phase 3 training. For example, participants were asked to choose which of cues $\mathrm{B}$ or L was more likely to cause outcome $\mathrm{o3}$, and (on a different trial) which of cues D or J was more likely to cause outcome 04 . The order of the test trials was randomized with one constraint: All of the test trials that tested the differences between the previously blocked target cues that were presented in new or old compounds were shown first.

\section{Results}

The same performance criteria from Experiment 1 were adopted for Experiment 2, and this resulted in the removal of 3 participants. The remaining participants' training data were very similar to those for the blocking contingencies of Experiment 1, showing rapid acquisition of the experimental contingencies in each training phase.

When we analyzed the choice data for each test trial, choice of the first item listed in Table 2 was scored as 1, and choice of the second item listed was scored as 0 . This means that, for the upper four test trial types of Table 2, each choice that favored competing cues over blocked target cues was scored as 1 . These choice scores were then averaged across these four equivalent trial types to yield a mean score between 0 and 1 , where 0 indicates consistent choice of the target cue and 1 indicates consistent choice of the competing cue. For the remaining four test trials listed in the lower section of Table 2, each choice that favored the previously blocked target cues presented in novel compounds over those presented in old compounds was scored as 1 ; these were again averaged to yield a mean score between 0 and 1 . For both mean scores, a value of .5 indicates chance performance, with participants equally likely to choose either option.

The choice tests of previously blocked target cues versus nonblocked competing cues yielded a mean choice score of $.69(S E M=.035)$. A one-sample $t$ test revealed that this was significantly different from chance $[t(30)=$ 5.34], indicating that participants reliably chose the competing cue over the target cue. In contrast, the choice tests between blocked target cues from old and new compounds yielded a mean choice score of $.52(S E M=.052)$, which did not differ significantly from chance $(t<1)$. Thus, we found no evidence that participants favored the target cues from either old or new compounds when they were forced to choose between them.

\section{Discussion}

We replicated the learned predictiveness effect observed in Experiment 1 in Experiment 2, using a forced choice testing procedure. When faced with a choice between a previously blocked target cue and a pretrained competing cue - both of which had been paired equally often with a particular outcome ( 03 or o4) during Phase 3 training participants reliably chose the competing cue as being more causal of that outcome. This finding is consistent with both conditional and unconditional views of attention. More important, it demonstrates that this paradigm, the choice test in particular, is sensitive to influences of learned predictiveness, legitimizing it as a test bed for comparing these views.

Contrary to the predictions of the conditional view of attention, participants did not endorse the target cues that were trained in new compounds during Phase 3 (cues L, $\mathrm{J}, \mathrm{P}, \mathrm{N}$ ) as being more causal than their counterparts that were trained in old compounds (cues B, D, F, H). Therefore, once again, no evidence was obtained for the operation of lateral inhibitory processes in attention. As for Experiment 1, these results are consistent with the view that learned biases in cue processing are not conditional on the presence of specific competing cues.

In summary, Experiments 1 and 2 provided two sources of evidence that conflict with a conditional account of attentional change in the blocking effect. First, in Experiment 1 , we observed a significant learned bias in attention away from previously blocked cues, even when those cues were later trained in novel compounds. This finding supports earlier research (Le Pelley et al., 2007; Le Pelley \& McLaren, 2003) suggesting that attentional changes cannot be driven solely by lateral inhibition from more predictive to less predictive cues, because the cues presented in these novel compounds had not been previously presented together, so there was no opportunity for a lateral inhibitory link to have formed between them. This evidence alone, however, does not constitute a serious challenge for existing formal models that include conditional attentional mechanisms (e.g., Kruschke, 2001, 2006), because, as noted earlier, these models typically include an unconditional attentional mechanism, too. Even a relatively simple dual-mechanism model that includes a simple unconditional attentional mechanism, in which the processing of a cue is influenced by the associative strength of that cue, can anticipate the attentional effects in novel compounds observed in Experiments 1 and 2. According to this account, a cue that has a strong association with an outcome will tend (all other things being equal) to command more attention than will a cue with a weaker association to the outcome. Therefore, within this simple dual-mechanism model, the bias in attention away from blocked cues in novel compounds may be explained as 
resulting from this simple unconditional mechanism of attentional allocation: As a consequence of the prior blocking treatment, blocked cues will begin Phase 3 with lower associative strength (and hence, lower unconditional attention) than competing cues.

The second source of evidence against a conditional account of attention is more problematic for two-mechanism accounts, such as that proposed by Kruschke $(2001,2006)$, because it suggests that at least in the present experiments, the decrement in attention for the blocked cue is entirely unaffected by a lateral inhibitory mechanism. Unlike in previous studies, we compared the magnitude of the decrement in learning for a previously blocked cue when it was later presented in the presence or absence of the cue that blocked it, and thus in the presence or absence of a possible lateral inhibitory link between those cues. In Experiment 1 , the magnitude of the decrement in learning for the previously blocked target cues was not influenced by whether those cues appeared in new or old compounds. To the extent that attention to the blocked target cue was conditional on the presence of the cue that blocked it, we would expect a greater decrement in learning for the blocked target cues presented in old compounds, relative to those presented in new compounds. However, no such effect was observed. Furthermore, in Experiment 2, participants did not demonstrate a preference between two previously blocked target cues-one that had been presented in compound with the competing cue that had previously blocked it, and one that had been presented in a novel compound with another competing cue. Thus, not only is a purely conditional account unable to explain the present results, it also adds no explanatory power to a purely unconditional account. That is, a model incorporating both a conditional and an unconditional mechanism for attentional allocation could explain these findings only by assuming that the contribution of the conditional mechanism was negligible.

There is, however, a possible reprieve for a conditional mechanism of attentional change (instantiated within a two-mechanism approach). Recall that, in Experiment 1, responding was generally higher to cues that were presented in the same compounds in Phases 2 and 3, as opposed to equivalent cues that were presented in novel compounds during Phase 3 . The most likely explanation of this finding rests with the formation of within-compound associations during training. Recall the basic $\mathrm{A}-\mathrm{o} 1, \mathrm{AB}-\mathrm{o} 1$, $\mathrm{AB}-\mathrm{o} 3$ design used for training of the old compounds. During Phase 2's AB-o1 training, participants could learn an association between cues $\mathrm{A}$ and $\mathrm{B}$, in addition to associations between the cues and the outcome. This A-B, within-compound association would be further reinforced on the $\mathrm{AB}-\mathrm{O} 3$ trials of Phase 3. Consequently, presentation of $\mathrm{B}$ (a weak predictor of $\mathrm{o} 3$ ) on test might retrieve a representation of $\mathrm{A}$ (a strong predictor of $\mathrm{o} 3$ ), and the influence of this retrieved representation could inflate participants' judgment of the causal efficacy of B. These within-compound associations and, hence, their influence on test ratings, would be greater for cues trained in old compounds during Phase 3, which were consistently paired in Phases 2 and 3, than for those trained in new compounds, which were paired only in Phase 3.

This raises the possibility that the influence of these within-compound associations masked the occurrence of a lateral inhibition effect. As indicated above, the cues that would gain most from within-compound associations were the previously blocked target cues presented in old compounds during Phase 3 (e.g., cue B), because these cues had strong associations to strong predictors of the Phase 3 outcome. One effect of this influence would be to increase the perceived causal efficacy of target cues from old compounds, relative to those from new compounds, directly opposing any influence of lateral inhibition on attention. Therefore, the absence of a difference between target cues from old and new compounds in Experiments 1 and 2 could be due to a genuine absence of lateral inhibition, or it could be due to the effect of within-compound associations masking any influence of lateral inhibition.

In this latter approach, in order to explain the lack of a significant difference between target cues from old and new compounds, it would be necessary to suppose that the influence of within-compound associations is at least as strong as that of lateral inhibition of attention. A third experiment was conducted to examine this assumption. In Experiment 3, we used a different set of control cues, based on those used by Kruschke and Blair (2000), which allowed us to examine the relative contributions of within-compound associations and of lateral inhibition between the pretrained competing cues and the blocked target cues.

\section{EXPERIMENT 3}

We designed Experiment 3 to determine whether within-compound associations between the target and competing cues in old compounds had masked any influence of lateral inhibition of attention between these cues. As can be seen in Table 3, the trial types experienced for the old compound and new compound contingencies were the same as for Experiment 2. Experiment 3 additionally included a new set of control contingencies modeled on those used by Kruschke and Blair (2000). In each of these control contingencies, an individual cue (e.g., Q) was consistently paired with an outcome (o1) during Phases 1 and 2. In Phase 3, this cue was then presented in compound with a novel target cue $(\mathrm{R})$, and this compound was paired with a novel outcome $(\mathrm{QR}-\mathrm{o} 3)$.

Testing was again by means of two-alternative forced choices. The test trials shown in Table 3 have been divided into four types.

Test Type 1 (A vs. F, C vs. H, E vs. B, G vs. D) had a choice between competing and target cues from old compounds. Both conditional and unconditional accounts of attention agree that participants will choose the competing cues as having greater causal strength.

Test Type 2 (I vs. P, K vs. N, M vs. L, O vs. J) had a choice between competing and target cues from new compounds. A purely conditional account of attention predicts that participants will not display any systematic preference 
between these cues, because training in novel compounds during Phase 3 releases them from any influence of lateral inhibition. However, an account incorporating an unconditional mechanism (acting either alone or in concert with a conditional mechanism) predicts that participants will more often endorse the competing cues as having greater causal strength than the target cues.

Test Type 3 (B vs. L, D vs. J, F vs. P, H vs. N) had a choice between target cues from old and new compounds. Two influences are potentially present in this comparison. Conditional accounts of attention anticipate that during Phase 3, learning about the target cues from old compounds (e.g., cue B) will be reduced by lateral inhibitory links from the competing cues (e.g., cue A) that had previously blocked them, relative to target cues that were trained in new compounds (e.g., cue L). Working against this influence is the possibility that the target cues from old compounds will have stronger within-compound associations to their Phase 3 competing cue partners than will target cues from new compounds. This influence of within-compound associations would tend to increase the perceived causal efficacy of the target cues from old compounds relative to those from new compounds, thus countering any influence of lateral inhibition. Hence (as argued earlier in the context of similar comparisons made in Experiments 1 and 2), from the point of view of a conditional approach to attention, this test type provides information on the relative strength of the influence of lateral inhibition and within-compound associations.

Test Type 4 (R vs. B, T vs. D, V vs. F, X vs. H) was adapted from Kruschke and Blair (2000) and had a choice between target cues from control contingencies and target cues from old compounds. From the point of view of a conditional model of attention, this test type should also index the relative influence of lateral inhibition and within-compound associations. During Phase 3, target cues from old compounds will be subject to lateral inhibition, but control cues will not, which will tend to favor control cues on test. However, these target cues from old compounds will also possess stronger within-compound associations to their competing cue partners, which will tend to favor cues from old compounds on test. Observing participants' choices for such compounds provides a second assessment of the relative strengths of these two influences.

\section{Method}

Participants and Stimuli. Experiment 3 was conducted with a new set of participants. Forty-one Cardiff University undergraduates participated in exchange for either course credit or a payment of $£ 5$. Stimuli were the same as for Experiment 1.

Procedure. The training procedure of Experiment 3 was the same as for Experiment 2, with the following exceptions: (1) Phase 1, Phase 2, and Phase 3 training now comprised 8 blocks, 6 blocks, and 8 blocks, respectively, and (2) there were no reminder blocks of Phase 1 trials during Phase 2. The test procedure for Experiment 3 was the same as for Experiment 2. In order to increase reliability, participants were presented with each of the choices listed in Table 3 twice. That is, after the participants had made each of the 16 choices in random order, the same 16 choices appeared again in random order. The order in which the two foods of each choice were presented on the screen (left/right) was counterbalanced across test blocks - for example, for choice "A vs. F," one presentation of the choice would have A on the left, and the other would have A on the right.

\section{Results}

The same performance criteria from Experiment 1 were adopted for Experiment 3, resulting in the removal of 1 participant. The remaining 40 participants' training data are shown in Figure 3. As in Experiments 1 and 2, participants showed rapid acquisition of the experimental contingencies in each training phase.

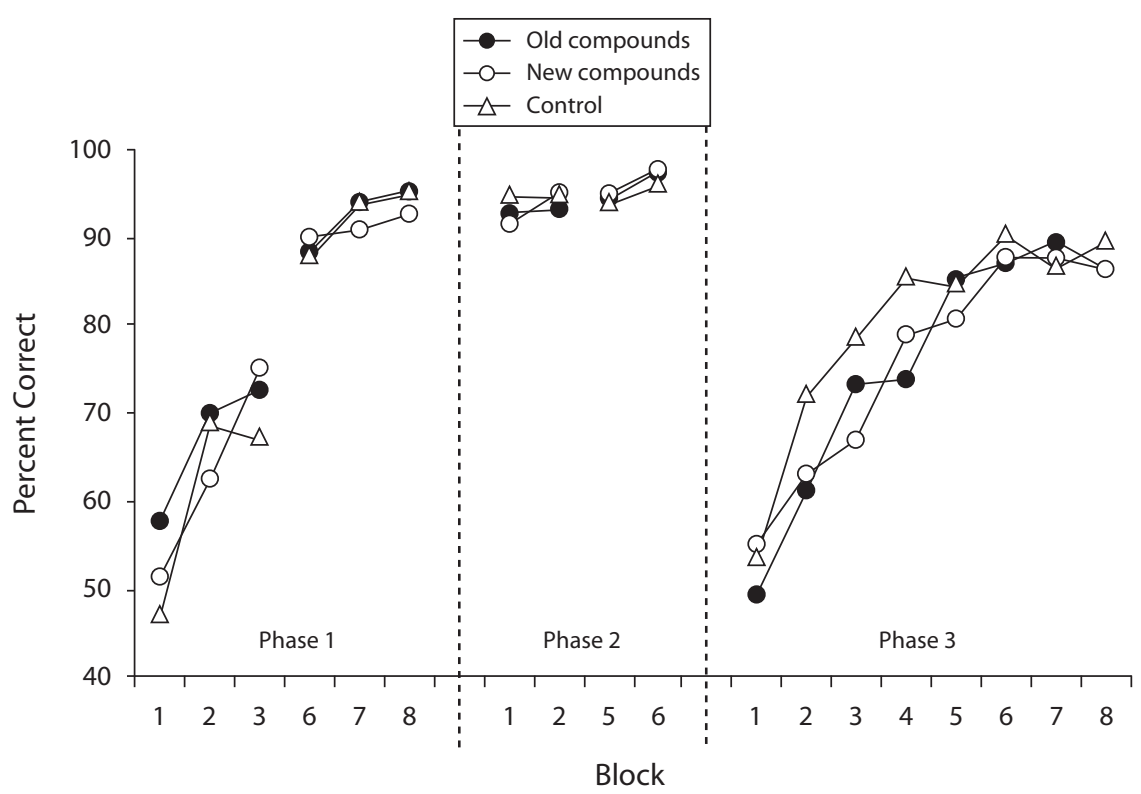

Figure 3. Mean percent correct responses for Experiment 3, showing the first and last three blocks of Phase 1, first and last two blocks of Phase 2, and all eight blocks of Phase 3. Data are averaged separately for old compounds, new compounds, and control contingencies. 


\begin{tabular}{|c|c|c|c|c|}
\hline \multicolumn{5}{|c|}{$\begin{array}{c}\text { Iable } 3 \\
\text { Design of Experiment } 3\end{array}$} \\
\hline \multicolumn{2}{|c|}{ Mr. X } & \multicolumn{3}{|c|}{ Mr. Y } \\
\hline \multirow[t]{2}{*}{ Phase 1} & \multirow[t]{2}{*}{ Phase 2} & Phase 3 & \multicolumn{2}{|c|}{ Test } \\
\hline & & \multicolumn{3}{|c|}{ Old Compounds } \\
\hline $\mathrm{A}-\mathrm{o} 1$ & $\mathrm{AB}-\mathrm{o} 1$ & $\mathrm{AB}-\mathrm{O} 3$ & \multirow{4}{*}{$1 \begin{array}{l}\text { A vs. F } \\
\text { C vs. H }\end{array}$} & \multirow{4}{*}{$\begin{array}{l}\text { Evs. B } \\
\text { G vs. D }\end{array}$} \\
\hline $\mathrm{C}-\mathrm{o} 1$ & $\mathrm{CD}-\mathrm{O} 1$ & $\mathrm{CD}-\mathrm{O} 4$ & & \\
\hline $\mathrm{E}-\mathrm{o} 2$ & $\mathrm{EF}-\mathrm{O} 2$ & $\mathrm{EF}-\mathrm{O} 3$ & & \\
\hline $\mathrm{G}-\mathrm{o} 2$ & $\mathrm{GH}-\mathrm{o} 2$ & GH-o4 & & \\
\hline \multicolumn{5}{|c|}{ New Compounds } \\
\hline & & & \multirow{4}{*}{$\begin{array}{l}\text { I vs. P } \\
\text { K vs. N }\end{array}$} & \multirow{4}{*}{$\begin{array}{l}\text { M vs. L } \\
\text { O vs. J }\end{array}$} \\
\hline $\mathrm{I}-\mathrm{O} 1$ & IJ-o1 & $\mathrm{IL}-\mathrm{o} 3$ & & \\
\hline $\mathrm{K}-\mathrm{ol}$ & $\mathrm{KL}-\mathrm{O} 1$ & $\mathrm{KJ}-\mathrm{O} 4$ & & \\
\hline $\mathrm{M}-02$ & $\mathrm{MN}-\mathrm{0} 2$ & MP-o3 & & \\
\hline $\mathrm{O}-\mathrm{o} 2$ & $\mathrm{OP}-\mathrm{o} 2$ & $\mathrm{ON}-\mathrm{O} 4$ & \multirow{2}{*}{$\begin{array}{l}\text { B vs. L } \\
\text { D vs. J }\end{array}$} & \multirow{2}{*}{$\begin{array}{l}\text { F vs. } \mathrm{P} \\
\mathrm{H} \text { vs. N }\end{array}$} \\
\hline & & Controls & & \\
\hline Q-o1 & Q-o1 & $\mathrm{QR}-\mathrm{o} 3$ & \multirow{4}{*}{$4 \begin{array}{l}\text { R vs. B } \\
\text { T vs. D }\end{array}$} & \multirow{4}{*}{$\begin{array}{l}\text { V vs. F } \\
\text { X vs. H }\end{array}$} \\
\hline $\mathrm{S}-\mathrm{ol}$ & $\mathrm{S}-\mathrm{o} 1$ & ST-o4 & & \\
\hline $\mathrm{U}-\mathrm{o} 2$ & $\mathrm{U}-\mathrm{o} 2$ & UV-03 & & \\
\hline $\mathrm{W}-\mathrm{0} 2$ & $\mathrm{~W}-\mathrm{0} 2$ & WX-o4 & & \\
\hline
\end{tabular}

Note-Numbers in bold refer to test trial types; see main text for explanation.

In analyzing choice data for each test trial, choice of the first item listed in Table 3 was scored as 1, and choice of the second item listed was scored as 0 . For each of the four test types $(1,2,3,4)$, these choice scores were averaged across the two presentations of each of the four corresponding choices, to yield a mean choice score between 0 and 1 , where 1 indicates consistent choice of the first item listed in Table 1 and 0 indicates consistent choice of the second item. A mean choice score of .5 indicates chance performance, with participants equally likely to choose either option. Mean choice scores for the four test types are shown in Figure 4. One-sample $t$ tests revealed that the mean choice score for each of the four test types was significantly greater than chance $[t(39)=3.37,5.03,2.19$, and 2.19, for Test Types 1, 2, 3, and 4, respectively].

\section{Discussion}

Test Type 1 demonstrated that for cues trained in old compounds, participants reliably chose competing cues as having greater causal strength than target cues. This is consistent with similar findings in Experiments 1 and 2 and again indicates that this paradigm is sensitive to influences of learned predictiveness.

Test Type 2 demonstrated that the same applied for cues trained in new compounds: Once again, participants were significantly more likely to choose competing cues as having greater causal strength than target cues. This echoes a similar effect in Experiment 1 and, as with that effect, lies beyond any cue-processing model that is based purely on conditional mechanisms of attention allocation. In contrast, this finding sits easily with a model in which attentional changes are not conditional on the presence of specific competing cues. If anything, the choice preference appeared stronger for cues from new compounds (Test Type 2) than for those from old compounds (Test Type 1). A paired $t$ test revealed that the difference between the mean choice scores in the two conditions approached significance $[t(39)=1.79, p=.080]$.

In line with this trend, the results for Test Type 3 indicated that participants were significantly more likely to choose the target cue from an old compound as having greater causal strength than that from a new compound. This is consistent with the finding of a general advantage for cues trained in old compounds in Experiment 1 and, as argued earlier, is most likely to reflect the influence of stronger within-compound associations for old compound cues. That said, the direct comparison of blocked target cues in Experiments 1 and 2 did not yield significant differences, and only Experiment 1 exhibited a numerical trend in the same direction as the significant effect found in the present experiment. This implies that the influence of within-compound associations was greater in Experiment 3 than in Experiments 1 and 2. There are several possible reasons for this, perhaps the most likely being

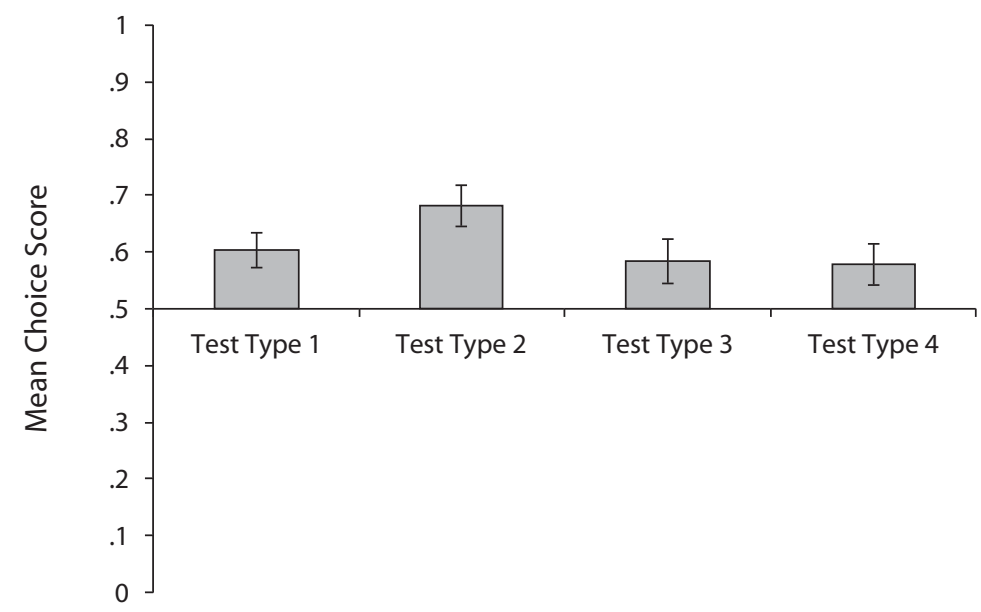

Figure 4. Mean choice scores for Experiment 3. A mean score of 1 indicates consistent choice of the first item listed in Table 1 across both presentations of all four exemplars of a particular test type, and 0 indicates consistent choice of the second item. 
the omission in Experiment 3 of reminder trials of Phase 1 training during Phase 2. These single-element reminder trials might be expected to weaken any within-compound associations in Experiments 1 and 2 through a process of extinction.

As argued previously, the finding of a significant learned predictiveness effect for novel compounds lies beyond any account of attention that is based purely on conditional processes; it can be captured by a two-mechanism model that combines conditional attention with a simple unconditional cue-processing mechanism that is based solely on the associative strength of cues. Such a model can then be applied to the comparison between target cues from old and new compounds tested by Test Type 3 . These cues must begin Phase 3 with equal associative strength and, according to this model, with equal "unconditional attention." Only the target cues from old compounds will also be suppressed by conditional attention (through a process of lateral inhibition), which will tend to lead to a lower perceived causal strength of these cues on test. This will, however, be counteracted by the stronger withincompound associations possessed by these cues. Consequently, in order for such a model to explain the advantage for target cues from old compounds over those from new compounds observed in Test Type 3, it would need to assume that the influence of within-compound associations in this experiment is significantly greater than that of conditional attention.

This assumption, however, is contradicted by the results of Test Type 4. Consistent with the findings of Kruschke and Blair (2000), participants were significantly more likely to choose target cues from the control contingencies (e.g., R) as having higher causal strength than previously blocked target cues from old compounds (e.g., B). These target cues would begin Phase 3 with similar associative strength and, according to the model outlined above, would have similar unconditional attention. If anything, this unconditional attention would be greater to $\mathrm{B}$ than to R. Given that R is a novel cue, it is bound to start Phase 3 with zero associative strength; to the extent that blocking is not complete, the associative strength of B at the outset of Phase 3 will be greater than zero. During $\mathrm{AB}-\mathrm{O} 3$ training, conditional attention to $\mathrm{B}$ will be suppressed by lateral inhibition from $\mathrm{A}$, which will tend to lead to a lower perceived causal strength of $B$ than of $R$ on test. This will, however, be counteracted by the A-B within-compound association, which will tend to lead to greater responding to $\mathrm{B}$ than to $\mathrm{R}$ (which, as a novel cue, does not possess any within-compound association at the outset of Phase 3). In order to explain the participants' choice of R over B, this account would need to suppose that the influence of conditional attention is significantly greater than that of within-compound associations. However, this is clearly incompatible with the results of Test Type 3, which, as argued above, require the opposite assumption. Consequently, it seems impossible for an account combining conditional attention with a simple unconditional attention mechanism that is based on associative strength to provide a coherent account of the results of Test Types 3 and 4.
This can be contrasted with a purely unconditional cueprocessing account, such as that offered by Mackintosh (1975), in which attentional changes are based on a comparison of the predictive abilities of simultaneously presented cues, but in which these changes are not specific to particular cue combinations. The fundamental prediction from such a model is that there will be no difference in attention to equivalent cues trained in old or new compounds. In order to explain the significant advantage for target cues from old compounds observed in Test Type 3, such an account would again need to appeal to the influence of within-compound associations. Given that on this view, these within-compound associations are the only factor distinguishing old from new compounds, this influence need only be relatively weak in order to produce an advantage for cues from old compounds in Test Type 3. Now consider the comparison of B to R in Test Type 4. $\alpha_{\mathrm{B}}$ will be considerably lower than $\alpha_{\mathrm{R}}$ as a consequence of B's being blocked during Phase 2, which will tend to lead to a lower perceived causal strength of $B$ than of $R$ on test. This will be counteracted to a certain extent by the influence of within-compound associations, but, as noted above, this influence need only be weak on this account; hence, it is quite possible for such a model to predict, overall, a lower causal strength for B than for R, as observed in Test Type 4.

\section{GENERAL DISCUSSION}

Across three experiments, participants demonstrated less learning about cues that had previously been blocked, as compared with learning about cues that had not, despite the fact that during the critical third learning phase, both types of cues were equally predictive of their respective outcomes. The observation that differences in the previously experienced predictiveness of cues are able to exert an influence on the rate of subsequent learning about those cues is consistent with the results of several recent studies of similar effects in human learning (e.g., Bonardi, Graham, Hall, \& Mitchell, 2005; Le Pelley et al., 2007; Le Pelley \& McLaren, 2003; Le Pelley, Oakeshott, \& McLaren, 2005). More specifically, this finding is analogous to the "blocking of unblocking" effect previously reported in both human learning (Kruschke \& Blair, 2000; Le Pelley et al., 2007) and animal conditioning (Mackintosh, 1978; Mackintosh \& Turner, 1971), wherein blocked cues are shown to be slower to enter into novel associations than are control cues.

Whereas our focus in this article has been on theories according to which attention to predictive cues is maintained at a higher level than that to nonpredictive cues (e.g., Kruschke, 2001, 2006; Mackintosh, 1975), prior demonstrations of blocking of unblocking have also been consistent with other theories that allowed for learned changes in cue processing. Perhaps most notable of these is the model of Pearce and Hall (1980), which takes a very different view of the mechanism underlying this effect. Briefly, according to Pearce and Hall, animals will devote more processing power to learning about stimuli whose predictive status is unclear (i.e., those that are followed by 
surprising outcomes) than to those whose predictive status is well established (i.e., those that are reliably followed by the same outcome).

In some sense, then, the Pearce-Hall (1980) model, where unreliable predictors will maintain a higher associability than reliable predictors, is the opposite of the Mackintosh and Kruschke models, where the reverse holds true. The Pearce-Hall model explains blocking in a standard $\mathrm{A}+, \mathrm{AB}+$ design as resulting from a reduction in $\mathrm{B}$ 's associability, which in turn reflects the fact that the outcome occurring on $\mathrm{AB}+$ trials is well predicted by the presence of $A$ at the outset of compound training. Consequently, this model is able to account for a retardation in subsequent learning about $\mathrm{B}$, relative to a control cue $\mathrm{D}$ that has been previously trained in a CD compound for which neither cue has been pretrained (so that the associability of $\mathrm{D}$ will be higher than that of B). This is the test used in all previously published studies of blocking of unblocking.

The Pearce-Hall (1980) model is unable, however, to account for the blocking of unblocking observed in the present experiments, which show retarded learning for a previously blocked cue, relative to the blocking cue with which it was previously trained. The model predicts that as a result of consistent pairings of $\mathrm{A}$ with an outcome during the pretraining phase of a blocking procedure, the associability of $\mathrm{A}$ will decline; hence, $\mathrm{A}$ will begin $\mathrm{AB}+$ compound trials with a lower associability than will $\mathrm{B}$. The original instantiation of the Pearce-Hall model predicts that during $\mathrm{AB}+$ training, B's associability will rapidly fall to exactly the same low level as A's. Pearce, Kaye, and Hall's (1982) later and more plausible refinement of this model predicts that the associability of B will approach that of A, but will remain higher. Neither version of the model is able to predict that the associability of B will fall below that of $\mathrm{A}$; hence, neither is able to explain the finding of slower subsequent learning about B than about $\mathrm{A}$. Consequently, it would seem that the general approach to attention offered by Mackintosh (1975) and Kruschke (2001, 2006), wherein attention is maintained for consistently predictive stimuli and declines for poorer predictors, is better able to explain blocking of unblocking - in human learning, at least.

Whereas the present experiments were clearly sensitive to the influence of a cue's prior predictiveness on subsequent cue processing, we found no evidence for conditional modulation of attention via a process of lateral inhibition, an idea that forms an important part of the general framework for attentional effects offered by Kruschke $(2001,2006)$. If present, such a process should result in decreased attention to the previously blocked cue B whenever it is presented with the cue A that initially blocked learning about it. Such effects should be reduced by removing cue A from the compound, hence removing any inhibition of attention. None of our experiments found evidence consistent with this prediction, even when controlling for the possible influence of within-compound associations (Experiment 3).

The present results are instead well explained by an unconditional account of attentional change, such as that proposed by Mackintosh (1975). According to this model, blocked cues lose associability (or attention) during the blocking procedure and are therefore subsequently less able to engage the learning process. Mackintosh's model suggests that the associability of a cue is independent of the cue with which it is paired. Hence, two cues with identical reinforcement histories will have the same attentional value, even if they are presented in novel compounds. In the present experiments, this view therefore predicts that the rate of learning about previously blocked cues will not depend on whether those cues are presented in new or old compounds, as was observed empirically.

The conclusion that the present data are supportive of an unconditional rather than conditional view of attention depends on our observations of the lack of any difference in the rate of learning between previously blocked cues embedded in old compounds as compared with those presented in new compounds. Therefore, a note of caution is required. Although the absence of evidence is not evidence of absence, the observation and replication of a strong and reliable blocking of unblocking effect in these experiments indicates that they were sensitive to influences of cue predictiveness on learning and, hence, presumably to differences in the magnitude of such influences. Furthermore, the choice testing procedure of Experiments 2 and 3 was used explicitly to maximize our chance of observing an advantage for previously blocked cues from new compounds over equivalent cues trained in old compounds. The motivation for this testing procedure was that if participants learned only slightly more about the previously blocked cues that were presented in new compounds, perhaps these differences were obscured by the requirement to individually rate different stimuli in Experiment 1 or were minimized by floor effects. Using a forced choice procedure, any small differences between the cues should have become apparent when participants were forced to choose between them, and the use of a relative, rather than absolute, test can reduce any potential impact of a response floor. However, once again this approach failed to find any evidence of greater suppression of target cues trained in old compounds, and was thus unsupportive of a conditional account of attention.

The observation in certain comparisons of an advantage for cues trained in old compounds over corresponding cues trained in new compounds in Experiments 1 and 3 led to the suggestion that within-compound associations could have influenced the responding to cues. That is, if cue $\mathrm{X}$ had a strong within-compound association to cue $\mathrm{Y}$, responding to cue $\mathrm{X}$ would be influenced by the strength of the Y-outcome association. These within-compound associations are most easily understood as associations between representations of cues.

How, then, do these examples of cue-cue learning differ from the cue-cue learning of lateral connections that lead to conditional changes in attention? The simple answer is that within-compound associations only influence responding to cues, whereas the lateral connections implementing conditional attention also lead to changes in the rate of learning about cues (i.e., in the attention paid to those cues). Thus, it may be possible for a single model to contain both a lateral inhibition mechanism and 
a mechanism that learns within-compound associations, but how these features could be implemented within specific models is a matter of speculation.

It was noted earlier that the results of Experiment 3 provide a serious challenge to a two-mechanism model combining conditional attention with a simple unconditional mechanism based on the associative strength of cues. Fundamentally, this is because such a model is constrained to predict equal or greater unconditional attention to a blocked cue (e.g., B) than to a novel control cue (e.g., R), which, when combined with analysis of participants' responding to the various test types, leads to a logical inconsistency regarding the relative strengths of conditional attention and within-compound associations. Our results need not, however, contradict a two-mechanism model, such as that proposed by Kruschke (2006), which combines conditional attention with an unconditional mechanism based on the relative predictiveness of cues - that is, with an unconditional mechanism based on the Mackintosh (1975) model. Such a model would predict less unconditional attention to a blocked cue than to a novel control cue, hence allowing it to provide a coherent account of the present results.

Consequently, the question becomes one of the extent to which it is necessary to invoke conditional attention mechanisms. As demonstrated earlier, a single-mechanism model of unconditional attention is able to provide a full account of our findings and is hence favored by parsimony. Moreover, the present experiments indicate that, at worst, conditional attention has no influence on learning, and, at best, the influence of conditional attention is significantly less than that of within-compound associations in these experiments. This is not to say, however, that the present experiments rule out the notion of conditional attention; they merely indicate that any contribution it might make to the blocking effect is minimized in these learning tasks. For example, it is possible that the large number of cue combinations used in each of the present experiments results in participants making less use of knowledge regarding particular combinations; studies using fewer combinations may be more likely to yield evidence for conditional effects of attention in blocking. To the best of our knowledge, however, there currently exists no compelling evidence providing an unequivocal demonstration of the conditionality of attention in learning. ${ }^{2}$

The extent to which the allocation of attention is conditional on the presence or absence of specific competing cues bears some relation to the issue of the context specificity of attention. The conditional approach to attention can be seen as highly context specific, where attention to a particular cue $\mathrm{X}$ is dependent on the context provided by other cues presented simultaneously with X. However, the converse need not be true: An unconditional account, as advocated in the present article, does not require that attention be context independent. Up to this point, our discussion has assumed that cues are represented as separable elements. However, the representation of a particular cue may include information about the context in which that cue is presented (see, e.g., Pearce, 1987, 1994). Within such a configural representation system, the allocation of attention would be influenced by the context in which a cue is presented, because the same cue presented in a different context would activate a slightly different representation, so that the attention developed to the original representation of this cue might not be fully generalized to this new representation. In support of this suggestion, there is some evidence that attention could act at the level of configurations of multiple cues (George \& Pearce, 1999). In this way, it is possible for this approach to predict a change in allocation of attention with a sufficiently large change in context, despite remaining entirely unconditional, in that attention "belongs to" a specific configuration of cues and is consequently independent of the presence of other cues.

Such a context-dependent, configural account is not consistent with the specific model of associative learning proposed by Mackintosh (1975), because this model assumes that cues are processed elementally. In fact, the formalization offered by Mackintosh has a number of serious limitations, particularly with regard to phenomenasuch as conditioned inhibition, a procedure that bears certain similarities to the change contingencies of Experiment 1 - that require a direct interaction between cues at the level of learning (see Le Pelley, 2004). Such issues are problematic for the specific instantiation suggested by Mackintosh, but they need not undermine the general approach to attention that his theory proposes. Indeed, Le Pelley (2004; see also Kruschke, 1996) has demonstrated how Mackintosh's formalization may be modified in order to overcome several of these limitations. It must be stressed that we are not advocating Mackintosh's model, nor any of the more recent extensions of it, as an all-encompassing theory of associative learning. This, after all, is not even how the theory was originally presented by Mackintosh. Our aim has simply been to demonstrate that there is some merit in the general, unconditional approach to attentional effects offered by this model. Our support is for the spirit of the model, not its specific instantiation.

In summary, no evidence was found to support the idea of conditional attentional inhibition of a blocked cue by the pretrained cue, despite explicit manipulations to maximize our experimental sensitivity to such an effect. The most parsimonious account of these results is that offered by an unconditional approach to attention, such as that proposed by Mackintosh (1975), wherein attention "belongs to" a specific cue and is independent of the presence of other cues.

\section{AUTHOR NOTE}

The research described in this article was conducted while the first author was a visiting researcher at the School of Psychology, Cardiff University, and was supported by a grant from the United Kingdom Economic and Social Research Council to the second author. Additionally, the authors thank Chris J. Mitchell for his valuable comments throughout the course of this research. Correspondence concerning this article should be addressed to O. Griffiths, School of Psychology, University of New South Wales, Sydney, NSW 2052, Australia (e-mail: ogriffiths@ psy.unsw.edu.au).

\section{REFERENCES}

Bonardi, C., Graham, S., Hall, G., \& Mitchell, C. (2005). Acquired 
distinctiveness and equivalence in human discrimination learning: Evidence for an attentional process. Psychonomic Bulletin \& Review, 12, 88-92.

DiCKINSON, A., \& BURKE, J. (1996). Within-compound associations mediate the retrospective revaluation of causality judgements. Quarterly Journal of Experimental Psychology, 49B, 60-80.

Dickinson, A., \& Mackintosh, N. J. (1979). Reinforcer specificity in the enhancement of conditioning by posttrial surprise. Journal of Experimental Psychology: Animal Behavior Processes, 5, 162-177.

George, D. N., \& Pearce, J. M. (1999). Acquired distinctiveness is controlled by stimulus relevance not correlation with reward. Journal of Experimental Psychology: Animal Behavior Processes, 25, 363-373.

Holland, P. C., \& Kenmuir, C. (2005). Variations in unconditioned stimulus processing in unblocking. Journal of Experimental Psychology: Animal Behavior Processes, 31,155-171.

Kamin, L. J. (1969). Predictability, surprise, attention, and conditioning. In B. A. Campbell \& R. M. Church (Eds.), Punishment and aversive behavior (pp. 279-296). New York: Appleton-Century-Crofts.

KrusChKe, J. K. (1996). Base rates in category learning. Journal of Experimental Psychology: Learning, Memory, \& Cognition, 22, 3-26.

KRUSCHKE, J. K. (2001). Toward a unified model of attention in associative learning. Journal of Mathematical Psychology, 45, 812-863.

KruschKe, J. K. (2006). Locally Bayesian learning with applications to retrospective revaluation and highlighting. Psychological Review, 113, 677-699.

KRUSCHKE, J. K., \& BLAIR, N. J. (2000). Blocking and backward blocking involve learned inattention. Psychonomic Bulletin \& Review, 7, 636-645.

Le Pelley, M. E. (2004). The role of associative history in models of associative learning: A selective review and a hybrid model. Quarterly Journal of Experimental Psychology, 57B, 193-243.

Le Pelley, M. E., Beesley, T., \& Suret, M. B. (2007). Blocking of human causal learning involves learned changes in stimulus processing. Quarterly Journal of Experimental Psychology, 60, 1468-1476.

Le Pelley, M. E., \& McLaren, I. P. L. (2003). Learned associability and associative change in human causal learning. Quarterly Journal of Experimental Psychology, 56B, 68-79.

Le Pelley, M. E., Oakeshott, S. M., \& Mclaren, I. P. L. (2005). Blocking and unblocking in human causal learning. Journal of Experimental Psychology: Animal Behavior Processes, 31, 56-70.

Le Pelley, M. E., Oakeshott, S. M., Wills, A. J., \& Mclaren, I. P. L. (2005). The outcome specificity of learned predictiveness effects: Parallels between human causal learning and animal conditioning.
Journal of Experimental Psychology: Animal Behavior Processes, 31, 226-236.

Mackintosh, N. J. (1975). A theory of attention: Variations in the associability of stimuli with reinforcement. Psychological Review, 82, 276-298.

Mackintosh, N. J. (1978). Cognitive or associative theories of conditioning: Implications of an analysis of blocking. In S. H. Hulse, H. Fowler, \& W. K. Honig (Eds.), Cognitive processes in animal behavior (pp. 155-175). Hillsdale, NJ: Erlbaum.

Mackintosh, N. J., \& Turner, C. (1971). Blocking as a function of novelty of CS and predictability of UCS. Quarterly Journal of Experimental Psychology, 23, 359-366.

Pearce, J. M. (1987). A model for stimulus generalization in Pavlovian conditioning. Psychological Review, 94, 61-73.

Pearce, J. M. (1994). Similarity and discrimination: A selective review and a connectionist model. Psychological Review, 101, 587-607.

Pearce, J. M., \& Hall, G. (1980). A model for Pavlovian conditioning: Variations in the effectiveness of conditioned but not of unconditioned stimuli. Psychological Review, 87, 532-552.

Pearce, J. M., Kaye, H \& Hall, G. (1982). Predictive accuracy and stimulus associability: Development of a model for Pavlovian learning. In M. L. Commons, R. J. Herrnstein, \& A. R. Wagner (Eds.), Acquisition (Quantitative Analyses of Behavior: Vol. 3, pp. 241-255). Cambridge, MA: Ballinger.

\section{NOTES}

1. According to the conditional view of attention, on the other hand, these reminder blocks should have little effect. Given that according to this account, attentional changes are specific to cue compounds, any changes in attention that occur on $\mathrm{M}-\mathrm{O} 1$ trials will not influence the conditional distribution of attention that participants learn to make for the $\mathrm{MN}$ compound.

2. Kruschke (2006) used a conditional attention mechanism in order to simulate the highlighting effect. However, our own computer simulations show that a model having only an unconditional learning mechanism (e.g., Mackintosh, 1975) can also demonstrate a highlighting effect, if $\alpha$ is assumed to be moderately high initially - for which there is some evidence (Le Pelley, Oakeshott, \& McLaren, 2005).

(Manuscript received October 17, 2007; revision accepted for publication June 5, 2008 .) 